\title{
Association between Shammah Use and Oral Leukoplakia-like Lesions among Adult Males in Dawan Valley, Yemen
}

\author{
Badr Abdullah Al-Tayar ${ }^{1 *,}$ Mon Mon Tin-Oo ${ }^{2 *}$, Modh Zulkarnian Sinor ${ }^{2}$, \\ Mohammed Sultan Alakhali ${ }^{3}$
}

\begin{abstract}
Background: Shammah is a traditional form of snuff dipping tobacco (a smokeless tobacco form) that is commonly used in Yemen. Oral mucosal changes due to the use of shammah can usually be observed in the mucosal surfaces that the product touches. The aim of this study was to determine the association between shammah use and oral leukoplakia-like lesions. Other associated factors were also determined. Materials and Methods: A cross sectional study was conducted on 346 randomly selected adult males. Multi-stage random sampling was used to select the study location. After completing the structured questionnaire interviews, all the participants underwent clinical exanimation for screening of oral leukoplakia-like lesions Clinical features of oral leukoplakia-like lesion were characterized based on the grades of Axéll et al (1976). Univariable logistic regression and multivariable logistic regression were used to assess the potential associated factors. Results: Out of 346 male participants aged 18 years and older, 68 (19.7\%) reported being current shammah users. The multivariable analysis revealed that age, non-formal or primary level of education, former shammah user, current shammah user, and frequency of shammah use per day were statistically associated with the presence of oral leukoplakia-like lesions [Adjusted odds ratio $(A O R)=1.03 ; 95 \%$ confidence interval $(\mathrm{CI}): 1.01,1.06 ; P=$ $0.006],(\mathrm{AOR}=8.65 ; 95 \%$ CI: $2.81,26.57 ; P=0.001),(\mathrm{AOR}=3.65 ; 95 \%$ CI: $1.40,9.50 ; P=0.008),(\mathrm{AOR}=12.99$; 95\% CI: 6.34, 26.59; $P=0.001)$, and (AOR= 1.17; 95\% CI: 1.02, 1.36; $P=0.026)$, respectively. Conclusions: The results revealed oral leukoplakia-like lesions to be significantly associated with shammah use. Therefore, it is important to develop comprehensive shammah prevention programs in Yemen.
\end{abstract}

Keywords: Shammah - smokeless tobacco - oral leukoplakia-like lesions - Yemen

Asian Pac J Cancer Prev, 16 (18), 8365-8370

\section{Introduction}

Shammah is a traditional smokeless tobacco (SLT) used in Yemen, with various varieties, including white, black, and gray powders locally known as Toombak. These varieties differ in terms of composition. Black and white shammah are made from powdered tobacco leaf, slaked lime, ash, oil, and other substances and flavors (Amer et al., 1985; Samman et al., 1998), whereas gray shammah (locally known as Toombak) is sun-dried powdered tobacco leaves mixed with ash. Shammah is usually placed in the lower buccal or labial vestibules (Salem, 1992).

The relationship between different types of tobacco smoking and oral health is clear; its negative effect on oral mucosal tissues has also shown (Loyha et al., 2012; Halawany et al., 2013; Hassona et al., 2014). In addition to tobacco smoking, smokeless tobacco has also evidently shown its effect on various oral tissues (Al-Attas et al., 2014; Tiwari et al., 2014). With regard to the effect of shammah on oral mucosal tissues, limited studies have been published so far, which revealed the association between shammah and oral mucosal lesions such as oral leukoplakia-like lesions. Scheifele et al. (2007) had shown a significant association between shammah use and development of oral leukoplakia among shammah users. Zhang et al. (2001) also revealed such association and concluded that as such, Shammah-induced oral leukoplakia-like lesions may be considered precancerous. The present study has been undertaken to evaluate the association between shammah use and oral leukoplakialike lesions. Other risk associated factors with oral leukoplakia-like lesions were also determined among adult males in Dawan valley, Yemen.

\section{Materials and Methods}

\section{Study design}

This cross-sectional study was conducted from June to November 2014 at the Al-Ebtesammah Dental Clinic in Dawan Valley, Yemen involving male residents, aged 18 years and above.

${ }^{1}$ Community Dentistry, ${ }^{2}$ Dental Public Health, School of Dental Sciences,Universiti Sains Malaysia, Kubang Kerian, Kelantan, Malaysia, ${ }^{3}$ Department of Periodontology, Faculty of Dentistry, Jazan University, Jazan, Saudi Arabia ${ }^{\star}$ Equal contributors *For correspondence:drmonmontinoo@gmail.com 


\section{Reference population}

The reference population of this study included all the male residents of Dawan Valley aged 18 years and above.

\section{Inclusion and Exclusion Criteria}

Inclusion criteria were Yemeni males aged 18 years and above, and those participants with at least two natural teeth in one sextant of the mouth, while exclusion criteria included smokers, Khat leaves chewers, participants diagnosed with diabetic mellitus and blood dyscrasia, those with immune-compromise diseases, undergoing periodontal treatment procedures, consuming drugs that affect periodontal status, mentally handicapped, and those with impaired hearing.

\section{Sampling method}

Dawan Valley was divided into three regions, namely, Layser, Laymen, and surrounding villages, which separately consist of approximately 10 villages. Among these regions, the study areas were selected by employing the probability multi-stage sampling method. Consequently, the Layser region, including its Morah, Sobaik, and Al-Dofah villages, were included in the study using simple random sampling approach. In this event, the simple random sampling approach was again adopted to determine the number of participants for this study.

\section{Calibration}

Prior to collecting data, a calibration procedure was performed on 20 participants to examine the oral leukoplakia-like lesion with oral and maxillofacial pathologist. Interrater reliability for the raters was determined as the following:

Kappa $=0.82(P<0.001)$ and 95\% CI: 0.60, 1.03.

\section{Research tools}

Structured interview questionnaire: The interview questionnaire was structured to incorporate the sociodemographic characteristics (age, family income, and education level), oral hygiene practices (daily tooth brushing, daily dental flossing, dental visits, and mouth rinse with water after shammah use), shammah use status (never, former, and current user), types of shammah, frequency of shammah use per day, duration of being a shammah user, and duration of shammah placement in the mouth per minute.

\section{Operational Definitions}

Oral Leukoplakia-like Lesion: Oral leukoplakia-like lesion was described as a lesion of the oral mucosa, characterized based on the grades of Axéll et al. (1976) as the following: Grade 1: A superficial lesion with a color similar to that of the surrounding mucosa and with slight wrinkling and no obvious thickening. Grade 2: A superficial whitish or yellowish lesion with wrinkling and no obvious thickening. Grade 3: A whitish-yellowish to brown, wrinkled lesion with the intervening furrows of a normal mucosal color; it exhibits obvious thickening. Grade 4: A marked, heavily wrinkled white-yellowish to brown lesion.

\section{Shammah use status}

This study illustrated the status of shammah use according to Sinalkar et al., (2012) as follows: Never shammah users were the participants who have never consumed shammah. Former shammah users were those individuals who have previously consumed shammah, but have stopped their consumption for at least a year, and current shammah users are those who have been consuming shammah either on a daily or occasional basis.

\section{Clinical oral examination}

As mentioned previously, this study clinically noted and classified the presence of oral leukoplakia-like lesion according to the grades specified by Axéll et al., (1976). The lesions were viewed under good lighting and gauze was used to dry the tissue before observation.

In this study, the oral mucosa was divided into 7 areas, namely, area A (from upper right third molar to upper right first premolar), area B (from upper right cuspid to upper left cuspid), area C (from upper left first premolar to upper left third molar), area D (from lower left third molar to lower left first premolar), area E (from lower left cuspid to lower right cuspid), area $\mathrm{F}$ (from lower right first premolar to lower right third molar) and area $\mathrm{G}$ (other areas). All these mucosal areas were examined, in which the highest (worst) grade for each subject was recorded. Any lesion that fell in between grades was recorded to the lesser one. When no oral leukoplakia-like lesion was identified, a zero grade was recorded.

\section{Statistical analysis}

The Statistical Package for Social Sciences (SPSS, IBM, and Chicago, USA version 20.0) and STATA version 8.0 were used for data entry and analysis. The descriptive analysis was presented as mean and standard deviations (SDs) for normally distributed numerical variables and frequency with percentage (\%) for categorical variables. Multiple logistic regression estimation was used between the dependent (oral leukoplakia-like lesion: yes/no) and independent variables or covariates by completing the below procedures: Data exploration and descriptive statistics, bivariable analysis (simple logistic regression), construction of preliminary model (variable selection), verification of interactions and multicollinearity, confirmation of the assumptions, interpretation of the final model, and presentation of results.

\section{Ethical considerations}

The protocol of this study was approved by the Ministry of Health and Population, Dawan branch in Yemen and was authorized by the Human Research Ethics Committee at Universiti Sains Malaysia. Ref No: (USM/ JEPeM/283.2. (6)/Amend.(01). FWA Reg. No: 00007718; IRB Reg. No: 00004494.

\section{Results}

A total of 346 adult males (18 to 68 years old) agreed to participate in this study. The mean age of the total participants was 34.3, with an SD of 12.29. The number of participants in each category based on their 
Association between Shammah Use and Oral Leukoplakia-like Lesions among Adult Males in Dawan Valley, Yemen

socio-demographic characteristics is shown in Table 1. Prevalence of shammah use was $8.7 \%$ and $19.7 \%$ among former and current shammah users, respectively. Meanwhile, the family income distribution indicated that $55.8 \%$ of the sample population receives more than Yemeni Rial (YER) 20,000 per month, whereas $44.2 \%$ of the population receives a lower income. Of the total number of participants, $41.3 \%$ has completed their secondary level of education, $30.3 \%$ is in primary level, $24.0 \%$ in tertiary level, and $4.3 \%$ in non-formal education level. In terms of the oral hygiene practices of the study population, $47.1 \%$ of the participants do not brush their teeth, $23.7 \%$ brush their teeth more than once a day, $15 \%$ uses toothbrush when necessary, and $14.2 \%$ uses toothbrush once a day.

With regard to dental flossing, this study determined that the majority of the participants $(93.9 \%)$ did not use dental floss for teeth cleaning, and only $6.1 \%$ of the participants used dental floss. This study also indicated that the majority of the participants $(53.5 \%)$ have never visited the dentist, $32.4 \%$ visits the dentist only when it is necessary, $31.3 \%$ occasionally go to their dentist, and only $0.9 \%$ regularly visit their dentist. Moreover, this study observed that most of the participants $(76.5 \%)$ rinse their mouth with water after using shammah, whereas $23.5 \%$ do not. Among the different kinds of shammah (white, black, and toombak), toombak is the only kind that is currently used by the shammah users included in this study.

Among the shammah users in this study, the mean (SD) usage of shammah per day is 9.6 (5.31) times, the mean (SD) duration of being a shammah user is 10.5 (6.40) years, and the mean (SD) of duration of shammah placement in the mouth is 12.5 (8.60) $\mathrm{min}$.

Table 2 reveals the distribution of participants based on the grades of oral leukoplakia-like lesion in relation to shammah user groups. By comparing the grades of oral leukoplakia-like lesion among the shammah user groups, this study realized that the prevalence of oral leukoplakia like-lesion grade 1 is higher in never shammah user group $(67.6 \%)$, followed by $17.6 \%$ and $14.7 \%$ in the current and former shammah user groups, respectively. This study also determined that the prevalence of oral leukoplakialike lesion grade 2 is higher in the current shammah user $(80.0 \%)$, followed by the former and never shammah users at $10.0 \%$ for both. With regard to oral leukoplakia-like lesion grades 3 and 4, none of the never shammah users were clinically examined (grade 3 or 4 ). The prevalence of oral leukoplakia-like lesions is higher in the current shammah user group (92.9\%) for grades 3 and (100.0\%) for grade 4 than those in the former shammah users at 7.1 $\%$ (grade 3 ) and $0.0 \%$ (grade 4 ).

Table 3 shows the significant factors associated with oral leukoplakia-like lesion in univariable analysis. These significant factors are age of the participants, family income (YER 20,000 or less), educational level (nonformal or primary and secondary level), tooth brushing per day (never), dental attendance (never and when necessary), former shammah use, frequency of shammah use per day and, duration of being a shammah user.

Table 4 indicates the results of multivariable logistic regression analysis for oral leukoplakia-like lesions and associated factors with the development of such disease. In terms of age, the participants with an increase in one year of age have an increase of $3 \%$ times the odds to have developed oral leukoplakia-like lesion when other

Table 1. Characteristics of Subjects among Adult Males in Dawan Valley, Yemen $(n=346)$

$\begin{array}{ll}\text { Variable } & \text { Frequency (\%) }\end{array}$

Socio-demographic characteristics

Age (year) 34.3 (12.29)*

Family income (monthly)

YER 20,000 or less

$153(44.2)$

YER more than 20,000

$193(55.8)$

Educational level

No formal education

$15(4.3)$

Primary education

Secondary education

$105(30.3)$

$143(41.3)$

Tertiary education

$83(24.0)$

Oral hygiene practice

Daily tooth brushing

Never

$163(47.1)$

When necessary

$52(15.0)$

Once a day

$49(14.2)$

More than once a day

$82(23.7)$

Flossing

Yes $21(6.1)$

No 325 (93.9)

Dental attendance

Never

$185(53.5)$

When necessary $112(32.4)$

Occasionally

$49(13.3)$

Regularly

$3(0.9)$

Mouth rinse with water after shammah use Yes

$52(76.5)$

No

$16(23.5)$

History of shammah use

Shammah use status

Never shammah user

$248(71.7)$

Former shammah user

$30(8.7)$

Current shammah user

68 (19.7)

Types of shammah

White

$0(0.0)$

Black

$0(0.0)$

Toombak

68 (19.7)

Frequency of shammah per day $9.6(5.31)^{*}$

Duration of being shammah user (in year) 10.5 (6.40)*

Duration of shammah placement in mouth (in minute) 12.5 (8.60)*

*Mean (SD)

Table 2. Distribution of Subjects According to the Oral Leukoplakia-like Lesion in Relation to Shammah Status

\begin{tabular}{llccccc}
\hline Variable & & \multicolumn{4}{c}{ Highest grade of lesion, n (\%) } \\
& & Grade 0 & Grade 1 & Grade 2 & Grade 3 & Grade 4 \\
\hline Shammah status & Never user & $222(83.5)$ & $23(69.6)$ & $3(10.0)$ & $0(0.0)$ & $0(0.0)$ \\
& Former user & $21(7.9)$ & $5(14.7)$ & $3(10.0)$ & $1(7.1)$ & $0(0.0)$ \\
& Current user & $23(8.6)$ & $6(17.6)$ & $24(80.0)$ & $13(92.9)$ & $2(100.0)$ \\
\hline
\end{tabular}


Table 3. Factors Associated with Oral Leukoplakia- like Lesion among Adult Males in Dawan Valley from Simple Logistic Regression

\begin{tabular}{|c|c|c|c|c|}
\hline \multicolumn{2}{|l|}{ Variable } & Crude OR (95\% CI) & Wald statistics (df) & P-value \\
\hline \multicolumn{5}{|l|}{ Socio-demographic } \\
\hline \multicolumn{5}{|l|}{ Characteristics } \\
\hline & Age & $1.03(1.01,1.05)$ & $9.21(1)$ & 0.002 \\
\hline \multirow[t]{2}{*}{ Family income } & More than 20,000 & 1 & & \\
\hline & 20,000 or less & $2.30(1.38,3.83)$ & $10.22(1)$ & 0.001 \\
\hline \multirow[t]{3}{*}{ Educational level } & Tertiary & 1 & & \\
\hline & Non and Primary & $16.71(5.75,48.56)$ & $26.77(1)$ & 0.001 \\
\hline & Secondary & $3.40(1.12,10.27)$ & $4.70(1)$ & 0.03 \\
\hline \multicolumn{5}{|l|}{ Oral hygiene practies } \\
\hline \multirow[t]{3}{*}{ Daily tooth brushing } & Once or more & 1 & & \\
\hline & Never & $2.69(1.48,4.91)$ & $10.52(1)$ & 0.001 \\
\hline & When necessary & $2.09(0.93,4.66)$ & $3.26(1)$ & 0.071 \\
\hline \multirow[t]{2}{*}{ Daily dental flossing } & Yes & 1 & & \\
\hline & No & $1.86(0.53,6.49)$ & $0.95(1)$ & 0.329 \\
\hline \multirow[t]{3}{*}{ Dental attendance } & Regularly and occasionally & 1 & & \\
\hline & Never & $4.50(1.49,13.54)$ & $7.16(1)$ & 0.007 \\
\hline & When necessary & $3.51(1.19,10.30)$ & $5.22(1)$ & 0.022 \\
\hline \multirow[t]{2}{*}{ Mouth rinse after shammah } & Yes & 1 & & \\
\hline & No & $1.06(0.32,3.56)$ & $0.01(1)$ & 0.914 \\
\hline \multirow[t]{3}{*}{ History of shammah use } & Never shammah user & 1 & & \\
\hline & Former shammah user & $3.65(1.51,8.82)$ & $8.34(1)$ & 0.004 \\
\hline & Current shammah user & $16.70(8.75,1.87)$ & $72.96(1)$ & 0.001 \\
\hline \multicolumn{2}{|c|}{ Frequency of shammah per day } & $1.17(1.02,1.36)$ & $4.94(1)$ & 0.026 \\
\hline \multicolumn{2}{|c|}{ Duration of being shammah user (in year) } & $1.07(0.98,1.17)$ & $2.35(1)$ & 0.125 \\
\hline \multicolumn{2}{|c|}{ Duration of shammah placement in mouth (in minute) } & $1.02(0.95,1.10)$ & $0.61(1)$ & 0.434 \\
\hline
\end{tabular}

Table 4. Factors Associated with Oral Leukoplakia- like Lesion among Adult Males in Dawan Valley Using Multiple Logistic Regression

\begin{tabular}{|c|c|c|c|c|c|}
\hline Variable & & $\begin{array}{l}\text { Crude OR }{ }^{\mathrm{a}} \\
(95 \% \mathrm{CI})\end{array}$ & $\begin{array}{l}\text { Adjusted } \mathrm{OR}^{\mathrm{b}} \\
\quad(95 \% \mathrm{CI})\end{array}$ & $\begin{array}{l}\text { Wald statistics } \\
\text { (df) }\end{array}$ & P- value ${ }^{b}$ \\
\hline \multicolumn{6}{|l|}{ Socio-demographic } \\
\hline Age & & $1.03(1.01,1.05)$ & $1.03(1.01,1.06)$ & $7.61(1)$ & 0.006 \\
\hline \multirow[t]{3}{*}{ Educational level } & Tertiary & 1 & 1 & & \\
\hline & Non and Primary & $16.71(5.75,48.56)$ & $8.65(2.81,26.57)$ & $14.19(1)$ & 0.001 \\
\hline & Secondary & $3.40(1.12,10.27)$ & $2.94(0.92,9.36)$ & $3.33(1)$ & 0.068 \\
\hline \multirow[t]{3}{*}{ History of shammah use } & Never shammah user & 1 & 1 & & \\
\hline & Former shammah user & $3.65(1.51,8.82)$ & $3.65(1.40,9.50)$ & $7.08(1)$ & 0.008 \\
\hline & Current shammah user & $16.70(8.75,31.87)$ & $12.99(6.34,26.59)$ & $49.21(1)$ & 0.001 \\
\hline Frequency of shammah per day & & $1.17(1.02,1.36)$ & $1.17(1.02,1.36)$ & $4.94(1)$ & 0.026 \\
\hline
\end{tabular}

${ }^{\mathrm{a} S i m p l e ~ l o g i s t i c ~ r e g r e s s i o n, ~}{ }^{\mathrm{B}}$ Multiple logistic regression; Backward step wise LR multiple logistic regression was applied; Multicolinearity and interaction term were checked and did not found; (overall correctly classified percentage (81.8\%) and area under Receiver Operating Characteristics (ROC) curve (82.9\%) were checked

variables were controlled. For subjects with different education levels, those who had non-formal or have completed their primary level of education were at 8.65 times higher odds to have developed oral leukoplakia-like lesion as opposed to those who have accomplished their tertiary level of education. With respect to shammah status, the participants who previously used shammah are at 3.65 times higher odds to have developed oral leukoplakialike lesion compared with those who have never used it. Meanwhile, the participants who are currently using shammah are at 12.99 times higher odds to have developed oral leukoplakia-like lesion than those who have never used it. The participants with an increase in one day of shammah use have an increase of $17 \%$ times the odds to have developed oral leukoplakia-like lesion.

\section{Discussion}

Shammah status is a major factor in the development of oral leukoplakia-like lesions in some Arabian countries (Scheifele et al., 2007; Al Agili and Park, 2013). Similarly, this study found an association between shammah use and these lesions after adjusting for socio-demographic characteristics and oral hygiene practice. In our study, the current and previous shammah usage was linked to the development of oral leukoplakia-like lesions. The risk for current shammah users to develop these lesions was 12.99 times higher than for non-users (AOR= 
Association between Shammah Use and Oral Leukoplakia-like Lesions among Adult Males in Dawan Valley, Yemen

12.99; 95\%CI: $6.34,26.59 ; \mathrm{P}=0.001)$. In addition, the risk for former shammah users was 3.65 times higher than that for non-users $(\mathrm{AOR}=3.65 ; 95 \% \mathrm{CI}: 1.40,9.50$; $\mathrm{P}=0.008)$ when other variables were controlled. AlAttas et al., (2014) indicated that the risk of developing premalignant lesions is 13.84 times in shammah users. Similarly, our findings show that the risk for current shammah users to develop oral leukoplakia-like lesions was 12.99 times higher than for non-shammah users when other variables were controlled. In another study, the relative risk for oral mucosal lesion development is significantly higher for smokeless tobacco users (betel quid chewing with tobacco) than for those who chew betel quid without tobacco. Lee et al., (2003) reported that the oral leukoplakia development risk for non-smokers and non-drinkers who chewed betel quid without tobacco was significant at 10 - to 15 -fold. Furthermore, this risk is lower than reported for those who ingested areca nut products containing tobacco $(\mathrm{OR}=17.4)$ (Hashibe et al., 2000).

The difference in risks for those who used areca nut products with and without tobacco implies that tobacco can have an added effect on oral leukoplakia. Our finding suggested that the risk of oral leukoplakia like lesion development was 12.99 times lower than that reported in a previous study $(\mathrm{OR}=17.4)$. This discrepancy can be ascribed to the variation in the ingredients of each type of smokeless tobacco. With regard to the frequency of daily shammah use, the duration of being a shammah user, and the duration of shammah placement in the mouth per minute, the severity of ST-related lesions was best predicted by the frequency of SLT use rather than by the annual duration of SLT use or according to each serving placed into the mouth. The presence and severity of lesions increased as the number of times a day in which a subject used SLT increased. Univariable and multivariable logistic regression analyses indicated that those who had been using SLT for a long time suffered severe lesions. This finding can be attributed to the increased frequency of SLT use at present rather than the long duration of use. This result was consistent with those of previous studies (Little et al., 1992).

The relation of detected oral leukoplakia-like lesions to socio-demographic factors was investigated in the present study. The multivariate analysis results showed that age is a significant risk factor for the development of oral leukoplakia-like lesions. Our finding is supported by the results of other studies, which indicated that age is a significant risk factor for the onset of oral mucosal lesions (Lin et al., 2001; GonUl et al., 2011; Al-Attas et al., 2014). Furthermore, Al-Attas et al. (2014) noted that low education level is a significant predisposing factor for the development of such lesions. Similarly, our findings suggest that those with a low education level were at higher risk to develop oral leukoplakia-like lesion than those with a high education level when other variables were controlled $(\mathrm{AOR}=8.65 ; 95 \% \mathrm{CI}: 2.81,26.57 ; \mathrm{P}=$ $0.001)$. This result indicates that a low education level may contribute to the unhealthy lifestyles that promote the onset of such lesions.

As per a simple logistic regression analysis, oral leukoplakia-like lesions were positively but insignificantly related to low family income in the final model. This finding contradicts than that of Al-Attas et al. (2014), who reported that the premalignant oral lesions were positively related to low income in Saudi Arabia. This result can be attributed to the fact that most of the participants (55.8\%) in our study earn high incomes. In fact, the results of the present study were similar to those obtained from studies conducted in Saudi Arabia and in the Republic of Yemen. These studies revealed that oral mucosal lesions were negatively related to oral health variables, such as brushing frequency and dental visits, when other variables were controlled (Scheifele et al., 2007; Al Agili and Park, 2013). The lack of association between oral hygiene practices and oral leukoplakia-like lesions may attribute to the fact that the study population uses traditional hygiene practices, such as miswak instead of modern oral hygiene practices such as tooth brush and dental floss.

\section{Acknowledgements}

The authors of this study wish to thank the staff of the Al-Ebtesammah Dental Clinic in Dawan valley, Yemen, especially Dr. Hussin Mubark Alhamdy who assisted the progress towards completion of this study.

\section{References}

Al-Attas SA, Ibrahim SS, Amer HA, et al (2014). Prevalence of potentially malignant oral mucosal lesions among tobacco users in Jeddah, Saudi Arabia. Asian Pac J Cancer Prev, 15, 757-62.

Al Agili D, Park H (2013). Oral health status of male adolescent smokeless tobacco users in Saudi Arabia. East Mediterr Health J, 19, 711-9.

Amer M, Bull C, Daouk M, et al (1985). Shamma usage and oral cancer in Saudi Arabia. Ann Saudi Med, 5, 135-41.

Axéll T, Mornstad H, Sundstrom B (1976). The relation of the clinical picture to the histopathology of snuff dipper's lesions in a Swedish population. J Oral Pathol, 5, 229-36.

GonUl M, GUl U, Kaya I, et al (2011). Smoking, alcohol consumption and denture use in patients with oral mucosal lesions. J Dermatol Case Rep, 5, 64-8.

Halawany HS, Jacob V, Abraham NB, et al (2013). Oral cancer awareness and perception of tobacco use cessation counseling among dental students in four Asian countries. Asian Pac J Cancer Prev, 14, 3619-23.

Hashibe M, Sankaranarayanan R, Thomas G, et al (2000). Alcohol drinking, body mass index and the risk of oral leukoplakia in an Indian population. Int $J$ Cancer, 88, 129-34.

Hassona Y, Scully C,Almangush A, et al (2014). Oral potentially malignant disorders among dental patients: a pilot study in Jordan. Asian Pac J Cancer Prev, 15, 10427-31

Lee C, Ko Y, Huang H, et al (2003). The precancer risk of betel quid chewing, tobacco use and alcohol consumption in oral leukoplakia and oral submucous fibrosis in southern Taiwan. Br J cancer, 88, 366-72.

Lin H, Corbet E, Lo E (2001). Oral mucosal lesions in adult Chinese. J Dent Res, 80, 1486-90.

Little SJ, Stevens VJ, LaChance PA, et al (1992). Smokeless tobacco habits and oral mucosal lesions in dental patients. J Public Health Dent, 52, 269-76.

Loyha K, Vatanasapt P, Promthet S, et al (2012). Risk factors for oral cancer in northeast Thailand. Asian Pac J Cancer 
Badr Abdullah Al-Tayar et al

Prev, 13, 5087-90.

Samman MA, Bowen ID, Taiba K, et al (1998). Mint prevents shamma-induced carcinogenesis in hamster cheek pouch. Carcinogenesis, 19, 1795-1801.

Scheifele C, Nassar A, Reichart P (2007). Prevalence of oral cancer and potentially malignant lesions among shammah users in Yemen. Oral Oncol, 43, 42-50.

Sinalkar D, Kunwar R, Bagal R (2012). Tobacco consumption and its association with education among women residing in a rural area of Maharashtra: A cross-sectional study. Med $J$ Armed forces india, 68, 335-8.

Tiwari RV, Megalamanegowdru J, Gupta A, et al (2014). Knowledge, attitude and practice of tobacco use and its impact on oral health status of 12 and 15 year-old school children of chhattisgarh, India. Asian Pac J Cancer Prev, 15, 10129-35.

Zhang X, Schmitz W, Gelderblom H, et al (2001). Shammahinduced oral leukoplakia-like lesions. Oral Oncol, 37, 609-12. 